\section{REFERENCES}

[1] R. A. Smith and J. M. Zias, "Comparison of the $\mathrm{p}$ - $\mathrm{n}$ fast switc ing rectifier and the Schottky rectifier," in Proc. IEEE/IAS Anizual Meet., pp. 60-68, 1976.

[2] J. R. Hauser and P. M. Dunbar, "Minority carrier reflecting pooperties of semiconductor high-low junctions," Solid-State E'lectron. vol 18, pp. 715-716, 1975.
[3] Y. Amemiya, T. Sugeta, and Y. Mizushima, "Novel low-loss and high-speed diode utilizing an ideal ohmic contact," IEEE Trans. Electron Devices, vol ED-29, pp. 236-243, 1982.

[4] D. J. Page, "Theoretical performance of the Schottky barrier power rectifier," Solid-State Electron. vol. 15, pp. 505-515, 1972.

[5] A. Nakagawa and M. Kurata, "Computer-aided design consideration on low-loss p-i-n diode," IEEE Trans. Electron Devices, vol. ED-28, pp. 231-237, 1981.

\title{
A Reliable Approach to Charge-Pumping Measurements in MOS Transistors
}

\author{
GUIDO GROESENEKEN, HERMAN E. MAES, NICOLAS BELTRÁN, AND ROGER F. DE KEERSMAECKER
}

\begin{abstract}
A new and accurate approach to charge-pumping measurements for the determination of the $\mathrm{Si}^{-} \mathrm{SiO}_{2}$ interface state density directly on MOS transistors is presented. By a careful analysis of the different processes of emission of electrons towards the conduction band and of holes towards the valence band, depending on the charge state of the interface, all the previously ill-understood phenomena can be explained and the deviations from the simple charge-pumping thury can be accounted for. The presence of a geometric component in s.sme transistor configurations is illustrated and the influence of trapping time constants is discussed. Furthermore, based on this insight, a new technique is developed for the determination of the energy distribution of interface states in small-area transistors, without requiring the $\mathrm{kn} \supset \mathrm{wl}$ edge of the surface potential dependence on gate voltage.
\end{abstract}

\section{INTRODUCTION}

$S_{\mathrm{d}}^{\mathrm{n}}$ NCE THE existence of surface states at the silicon/silicon dioxide interface was demonstrated, several technicues have been proposed for the determination of the density of these states and of their energy distribution in the forbidden energy gap of silicon.

Most of these techniques are based on measurements on MOS capacitors [1]- [4] and have been studied in great detail. Consequently they have become sufficiently reliable tc be used routinely in most laboratories. For the determination of the surface-state densities directly on MOS transistors only a few techniques are available; they are not commonly used, however, partly because of a poor quantitative reliability and partly because they are not very practical to use. Therefore none of these techniques can be qualified as generally accejted for MOS transistors.

A first method developed for MOS transistors extract: in formation on the surface states from the transistor behavior in the weak inversion region. Although satisfactory results were obtained using this technique it is only applicable for

Manuscript received April 6, 1983; revised August 2, 1983.

The authors are with the ESAT Laboratory, Katholieke Univer iteit Leuven, Kardinaal Mercierlaan 94, B-3030 Heverlee, Belgium. long-channel devices $(>20 \mu \mathrm{m})$ and at low drain voltages [6].

The deep-level transient-spectroscopy technique (DLTS) which was developed for capacitors can also be performed on transistors [7]. This technique yields information on surfacestate densities and captures cross sections from the measurement of capacitance transients resulting from electron and hole emission from the states to the conduction and the valence band. However, this set-up requires a high-quality averager to detect the small capacitance transients, and the analysis is rather complex.

The reported relation between $1 / f$ noise and surface-state density [8], [9] has been used in some cases to determine this surface state density. However, in view of the lack of agreement among the different theories on $1 / f$ noise such a determination is only qualitative.

And finally, we want to discuss the charge-pumping technique which was introduced by Brugler and Jespers [10]. This technique is based on a recombination process at the $\mathrm{Si} / \mathrm{SiO}_{2}$ interface involving the surface states. This induces a substrate current which can be directly related to the surface-state density. In spite of its capability for measurements on small-area MOST devices, it never became a standard technique because of some ill-understood phenomena which made the method qualitative rather than quantitative. Despite some separate attempts to explain these phenomena [10]-[15], a general model for the charge-pumping mechanism was not available until now. In this paper we will present a model which describes and explains all of the poorly understood phenomena taking into account the emission of holes and electrons to the valence band or the conduction band, respectively, depending on the state of $\mathrm{Si}_{-} \mathrm{SiO}_{2}$ interface. This model is completely confirmed by the experiments. By applying the technique in an adapted way it even becomes possible to obtain the energy distribution of the interface states from a very simple measurement, without knowing the dependence of the surface potential on the applied gate voltage [5]. 
The new insight in the underlying mechanisms of charge pumping makes this technique into a powerful and reliable quantitative method for determining interface-state densities, even on small-area MOS transistors. The simple standard equipment which is required for this technique makes it readily accessible for each investigator and moreover, because of the very short measuring times, it is extremely well suited for process evaluation, for example with wafer mapping.

First, in Section II the basic principles of the charge-pumping technique will be reviewed and the different proposed measuring procedures will be compared. Next, the problem areas and the poorly understood effects inherent to the charge-pumping technique will be indicated in Section III. In Section IV a reliable model for the charge pumping process will be presented. The experimental results will be described in Section $V$ and compared with the model predictions. Finally, in Section VI a new technique based on the new insight into the chargepumping process will be presented which allows the determination of the energy distribution of the interface states even on small-area MOS transistors.

\section{The Charge-Pumping Technique-Basic Principle}

The basic experimental set-up, as introduced by Brugler and Jespers [10], is illustrated in Fig. 1 in the case of an n-channel transistor. The source and drain of the transistor are connected together and held at a certain reverse bias voltage with respect to the substrate. When the transistor is pulsed into inversion, the surface becomes deeply depleted and electrons will flow from the source and drain regions into the channel, where some of them will be captured by the surface states. When the gate pulse is driving the surface back into accumulation, the mobile charge drifts back to the source and drain under the influence of the reverse bias, but the charges trapped in the surface states will recombine with the majority carriers from the substrate and give rise to a net flow of negative charge into the substrate. This is the so-called charge-pumping effect. The charge $Q_{s s}$ which will recombine is given by

$$
Q_{s s}=A_{G} \cdot q \int D_{i t}(E) d E .
$$

It can also be expressed as

$$
Q_{s s}=A_{G} \cdot q^{2} \cdot \overline{D_{i t}} \cdot \Delta \psi_{s}
$$

where

$A_{G} \quad$ is the channel area of the transistor $\left(\mathrm{cm}^{2}\right)$,

$D_{i t}(E)$ is the surface-state density at energy level $E\left(\mathrm{~cm}^{-2}\right.$. $\mathrm{eV}^{-1}$ ),

$\overline{D_{i t}}$ is the mean surface-state density, averaged over the energy levels swept through by the Fermi level $\left(\mathrm{cm}^{-2} \cdot \mathrm{eV}^{-1}\right)$

$\Delta \psi_{s} \quad$ is the total sweep of the surface potential, and

$q$ is the electron charge $(C)$.

When applying repetitive pulses to the gate with frequency $f$, this charge $Q_{s s}$ will give rise to a current in the substrate given by

$$
I_{C P}=f \cdot Q_{s s}=f \cdot A_{G} \cdot q^{2} \cdot \overline{D_{i t}} \cdot \Delta \psi_{s}
$$

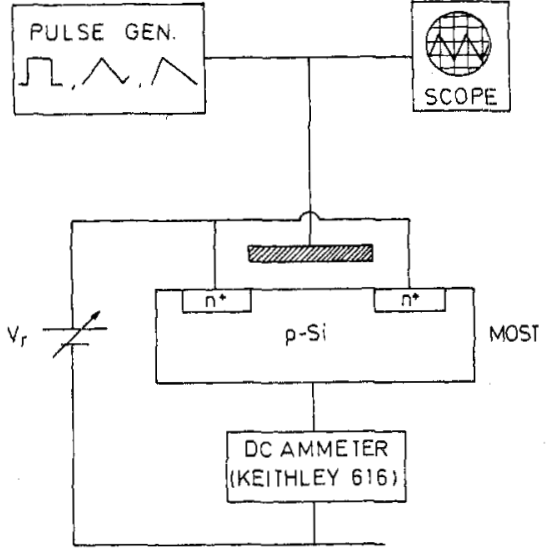

Fig. 1. Basic experimental set-up for charge-pumping measurements.

By measuring this substrate current, an estimate of the mean value of the interface-state density over the energy range swept by the gate pulse can be obtained.

Charge-pumping measurements have been performed in dif. ferent ways:

method A: by keeping the pulse base level in accumulation and pulsing the surface into inversion with in creasing amplitudes [10];

method B: by varying the pulse base level from inversion to accumulation while keeping the amplitude of the pulse constant [11]; and

method $\mathrm{C}$ : by keeping the pulse base level in inversion and pulsing the surface into accumulation with increasing amplitude [12].

In method $\mathrm{A}$ a saturation level for the charge-pumping current $I_{C P}$ is expected when the top of the gate pulse exceeds the threshold voltage of the MOS transistor. This saturation level corresponds to the value of the charge pumping current, given by (3) for a maximum value of $\Delta \psi_{s}$ and yields the number of interface states over this range, assuming all other parameters to be known. The work of [10] has been extended by one of the authors of the method [13]. A second increase in the chargepumping current was shown to be a contribution from the field region under the bonding area of the gate, and the loga. rithmic dependence of the current saturation level on the length of the gate pulse was suggested to be the result of the capture of carriers by oxide traps close to the interface due to a tunneling mechanism.

Method B was introduced by Elliot [11], in order to also determine an energy distribution of interface traps. This measurement yields three regions, depending on the position of the pulse base level $V_{G L}$. Only the second region (with $V_{T}-\Delta V_{G}<V_{G L}<V_{F B}$ where $V_{T}, \Delta V_{G}$, and $V_{F B}$ represent the threshold voltage, the pulse amplitude, and the flatband voltage, respectively) gives the charge-pumping current as determined by (3). Elliot derives the energy distribution from the rising edge of the current-voltage curve, when the pulse base level is going from inversion to accumulation, while the pulse top level is still in inversion. In this paper however, we will show that the variation of the charge-pumping current in this region cannot be used to determine the energy distribu- 


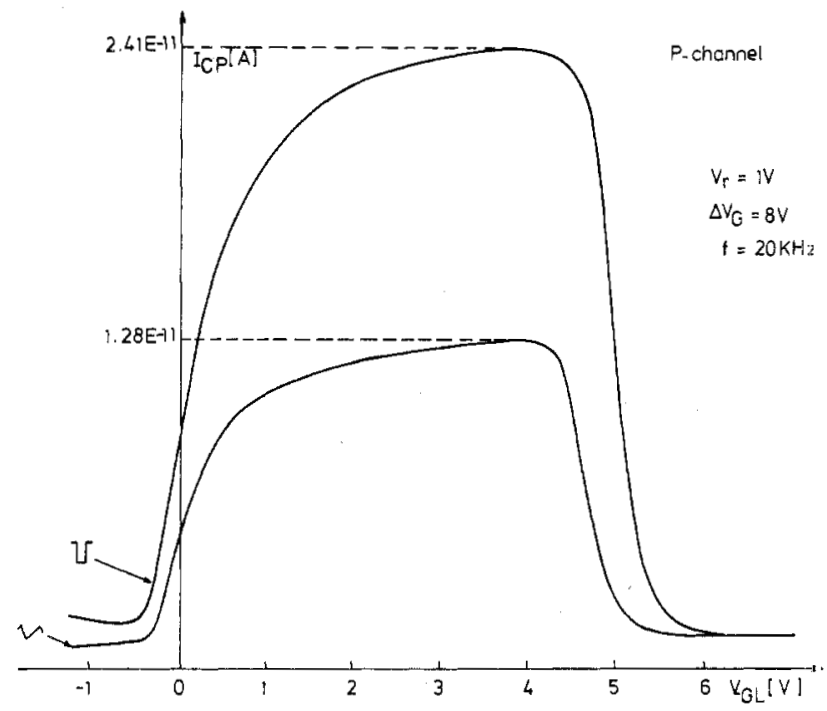

Fig. 2. Influence of pulse shape on the charge-pumping current wlien using method $\mathrm{B}\left(I_{C P}\right.$ as a function of gate pulse base-level $\left.V_{G L}\right)$.

tion of the interface states essentially for the same reason :"or which the rising edge in method $\mathrm{A}[10]$ could not be used ior this purpose.

In method C, finally, [12] the pulse base level is kept constant in inversion in order to avoid the so-called geometric component of the current, or at least to keep it constant. However, this goal is not reached because of several ot $\xi_{\text {ler }}$ mechanisms which are occurring and which will be described in Section IV. The geometric component refers to an aditional substrate current when some fraction of the mobile channel charge does not drift back to source and drain [10].

\section{Poorly Understood Phenomena}

The charge-pumping technique, as described in the previous section, has been used to determine the interface-state density in the silicon forbidden energy gap from a single substrete current measurement in an MOS transistor. As stated in the introduction, however, it never became a standard technique in spite of its attractiveness for measurements on small-area transistors because of some phenomena which were never wall explained. In this section we will list some of these problerns and mention the efforts which were undertaken by different investigators in order to explain these phenomena separately.

\section{A. Dependence on the Pulse Shape}

From the very first experiments on charge pumping, a stro: 19 dependence of the charge-pumping current on the shape of the applied gate pulses was observed [10], [14]. In Fig. 2 th is effect is illustrated when using method $B$ on one of our cevices. The first curve on this figure is obtained when using square pulses, the second when using sawtooth pulses. T.1e transistor under test was a p-channel device with a chanrel length of $6 \mu \mathrm{m}$ and a channel width of $30 \mu \mathrm{m}$. The pulse frequency was $20 \mathrm{kHz}$ and the reverse voltage was equal to $1 \mathrm{~V}$, which should be sufficient to eliminate any geometic component in the current. It is obvious that, even in th is short-channel transistor, a strong influence of the pulse shape on $I_{C P}$ is observed.

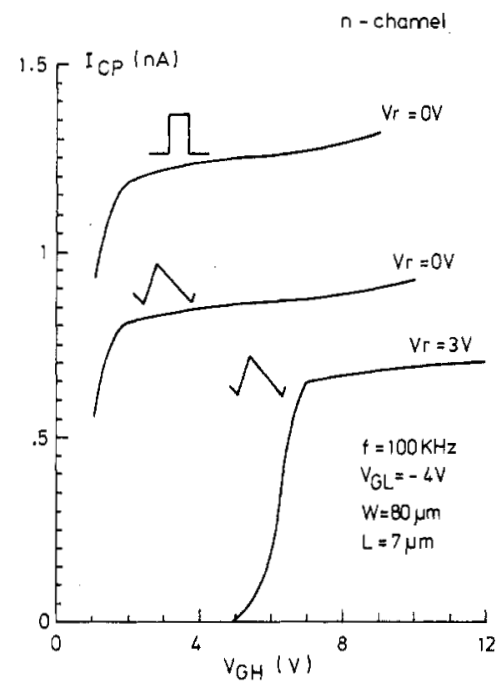

Fig. 3. Current increase as a function of the top level of the gate pulse for different measurement conditions, using method $\mathrm{A}$.

This phenomenon has been explained in the past by a geometric component [10], consisting of free minority carriers which do not have enough time to flow back to source and drain and are recombining with majority carriers and therefore also contribute to the charge-pumping current. It was believed that by using sawtooth pulses this component could be eliminated because of the longer time available for the mobile carriers to reach source and drain when driving the surface back towards accumulation. This was, however, not confirmed experimentally, as the charge-pumping current is a continuously decreasing function of the pulse fall and rise times, whereas a saturation would be expected for any fall and rise time larger than a few microseconds.

\section{B. Current Increase with Increasing Gate Voltage}

When using method $\mathrm{A}$ one expects a saturation of the current for sufficiently large gate amplitudes because the surface potential is pinned at $2 \phi_{F}$ once the channel is in inversion. However, one always observes a small but consistent current increase for increasing gate voltage amplitude [10] (Fig. 3).

This phenomenon was initially again explained by the geometric component. If some fraction $\alpha$ of the mobile charge does not drift back to source and drain, this part of the mobile carriers which can be expressed as

$$
Q_{\mathrm{mob}}=\alpha \cdot C_{\mathrm{ox}}\left(V_{G}-V_{T}\right)
$$

is recombining with majority carriers. The total substrate current is then given by

$$
I_{C P}=f \cdot A_{G} \cdot\left[q^{2} \cdot \overline{D_{i t}} \Delta \psi_{s}+\alpha C_{\mathrm{ox}}\left(V_{G}-V_{T}\right)\right] .
$$

It was believed that this geometric component could be eliminated by using sawtooth pulses as mentioned before, by using a reverse voltage at drain and source or by using method $\mathrm{C}$ mentioned in Section II. In the latter case, the geometric component was expected to be nonzero but constant because the inversion level of the pulse $V_{G H}$ is constant. However from Fig. 3 it is obvious that the substrate current still increases even when a reverse bias voltage or sawtooth pulses are used. Fig. 4 shows a comparison of the charge pumping currents for 


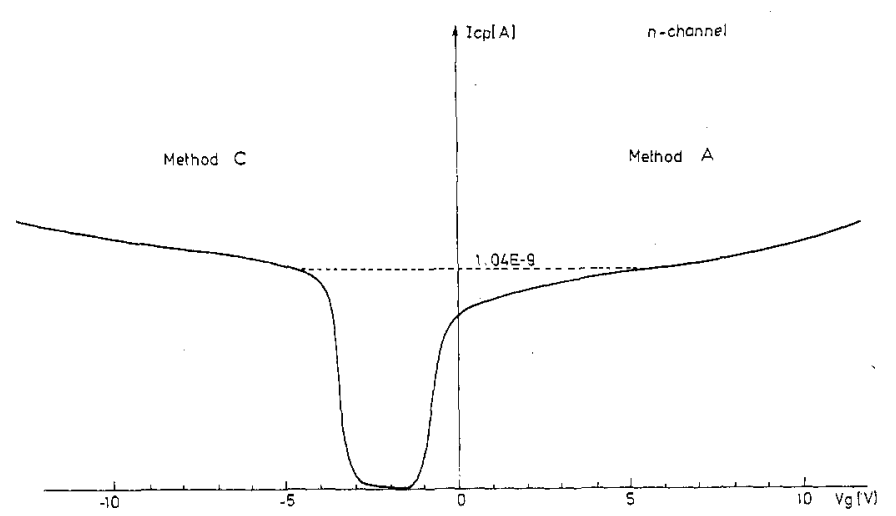

Fig. 4. Current increase with top level (method A) and base level (Method C) of the gate pulse, respectively.

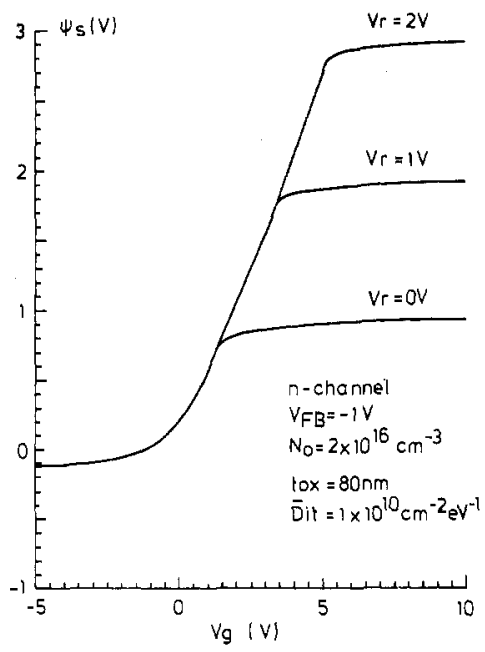

Fig. 5. Calculated dependence of surface potential on gate voltage for different reverse voltages at source and drain.

method $\mathrm{A}$ and method $\mathrm{C}$. It is clear that in neither case a saturation is obtained.

Another explanation for the current increase could be sought in the dependence of the surface potential on gate voltage. This surface potential is indeed not exactly pinned at $2 \phi_{F}$ when increasing the gate voltage, as is usually assumed, but is slightly dependent on the gate voltage in inversion. Fig. 5 shows this dependence for different reverse voltages $V_{r}$. One can see that once in inversion the surface potential still slightly increases. This means that for increasing gate voltage amplitudes the energy range over which the interface states are contributing to the substrate current slowly increases and so would also the substrate current in view of the previous interpretation of the charge-pumping mechanism.

However, as will become clear in Section IV, the surface potential does not play an important role in the actual value of the charge-pumping current, and therefore this explanation is irrelevant.

\section{Nonlinear Frequency Dependence for Triangular Pulses}

According to (3) a linear dependence of the charge-pumping current on the frequency $f$ is expected. This is indeed observed when using square pulses [10], but when sawtooth or triangular pulses are used, a nonlinear frequency dependence

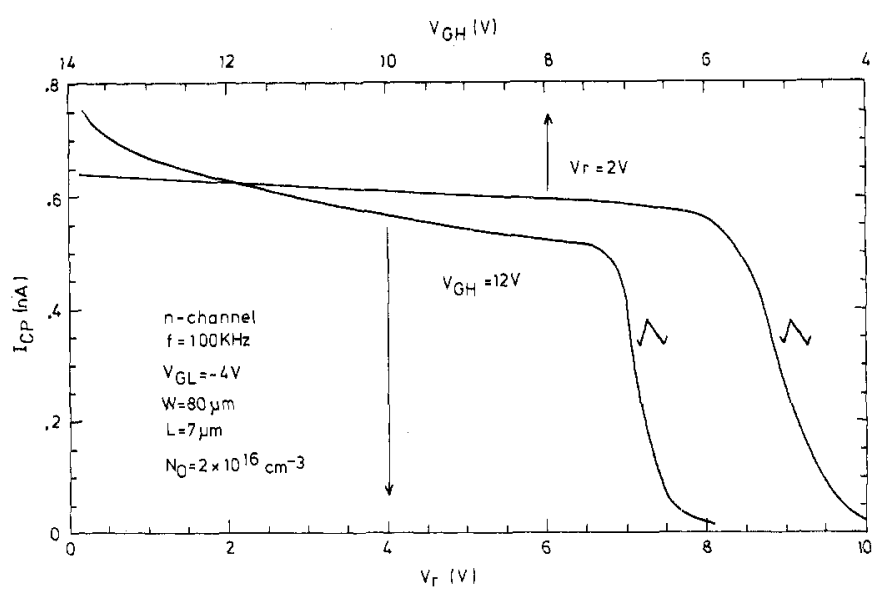

Fig. 6. Charge-pumping current as a function of top level of the gate pulse (upper scale) and of reverse voltage at source and drain (lower scale).

is observed. Different explanations for this phenomenon were proposed. Brugler and Jespers [10] tried to explain it by the maximum trapping time constant, but did not succeed in giving a quantitative description. Backensto and Viswanathan [15] claimed that this nonlinear relationship could be explained by a trap distribution with decreasing concentration into the oxide. However, their argumentation starts from an expression which is only valid for small signal responses and if thermal equilibrium occupancy functions hold. In the case of charge pumping, however, one does not deal with small signals and certainly not with thermal equilibrium. Moreover, the traps which are located deeper into the oxide will interact as long as they can be filled by inversion carriers. Therefore, the time that the surface is inverted should be larger than the trapping time constant of these traps. When this condition is no longer fulfilled, they will not contribute to the current anymore. This phenomenon has already been described by Declercq and Jespers [13]. Furthermore, the nonlinear relationship obviously is not observed when applying rectangular pulses, while the explanation based on oxide traps does not take the pulse shape into account.

\section{Current Decrease with Increasing Reverse Voltage}

By applying a reverse voltage at source and drain, the chargepumping current is observed to decrease [10], [11], [13]. On Fig. 6 the charge-pumping current is plotted as a function of the reverse voltage at source and drain, while the gate pulse is kept at a constant amplitude and base level. In the same figure the normal charge-pumping characteristic is also shown for increasing gate pulse amplitudes, and a constant reverse voltage at source and drain. The two curves differ mainly in two ways.

First the current step towards saturation is occurring for a different $\left(V_{G H}-V_{r}\right)$ value. For the curve with variable reverse voltage, this step occurs at about $12 \mathrm{~V}-7 \mathrm{~V}=5 \mathrm{~V}$, while for the one with variable gate amplitude it occurs at $5 \mathrm{~V}$ $2 \mathrm{~V}=3 \mathrm{~V}$. This difference can be simply explained by the body effect on the threshold voltage. For the curve with constant reverse voltage, this influence is constant, while for the other one, the body-effect changes with the applied reverse voltage. 
The second difference between the two curves is the level at which the current saturates. For the variable gate ampl tude curve, it saturates at a higher level, but the subsequent increase is less than for the variable reverse voltage curve. This behavior can be explained by the modulation of the effective area under the gate where the recombination process occurs as a rest.lt of the widening of the surface space-charge layers around sciurce and drain with increasing reverse voltage during the accur nulation period. However, when one tries to predict this molulation with a simple one-dimensional model, one obtains a surface doping concentration which is about 50 percent lower than the one obtained with other methods for the deternination of the surface doping concentration.

In the case of Fig. 6 one needs a surface doping of less than $1 \times 10^{16} \mathrm{~cm}^{-3}$ in order to explain the difference betweer: the two saturation levels. However, the surface doping concentration obtained by conventional measurement techniques (e.g., body effect) was found to be $2 \times 10^{16} \mathrm{~cm}^{-3}$. This means that the charge-pumping current shows a more rapid decrease 'with increasing reverse voltage than could be expected from the one-dimensional model. Declercq and Jespers [13] tried to explain this phenomenon by assuming an increase in the interface state density in the vicinity of the junctions due to the presence of impurities or dislocations associated with the diffusion mechanism of source and drain.

\section{A Reliable Model for Charge Pumping}

In order to understand all the phenomena mentioned in the previous section, one has to consider in more detail the different mechanisms which are occurring when applying pulses at the gate. In this derivation we assume that no geometric component is present during the experiment which will be confirmed later on. Brugler and Jespers [10] already showed that this condition is normally fulfilled if the geometry of the t:ansistor is such that $W / L \gg 1$.

When the transistor is switched from accumulation towards inversion and vice versa, the state of the channel of the MOSFET goes through three different modes, each of wh ich can be characterized by a different time constant and escentially corresponds to one of the conventional operating regions of an MOS structure (i.e., accumulation, depletion, and inversion). Let us consider a $p$-type material ( $n$-channel tiansistor). A waveform as shown in Fig. 7 is applied to the cate of the transistor. It has a rise time $t_{r}$ and a fall time $t_{f}$, an amplitude $\Delta V_{G}=V_{G H}-V_{G L}$, and a period $T_{p}$. Fig. 8 shows a schematic representation of the different processes which are operating during one cycle. When the surface is in accumulation ( $V_{G}$ negative), all of the surface states below the quasi-Fermi level of the minority carriers are filled with eectrons, while those above it are empty. The states are thus in equilibrium with the energy bands.

1) When the gate voltage increases, the surface potential is changing at a certain rate. Therefore, holes that have to be emitted from the states towards the valence band (which in fact are electrons from the valence band emitted to the stales) in order to maintain equilibrium, will flow back to the s sbstrate. Initially, the rate of emission of trapped charge is able

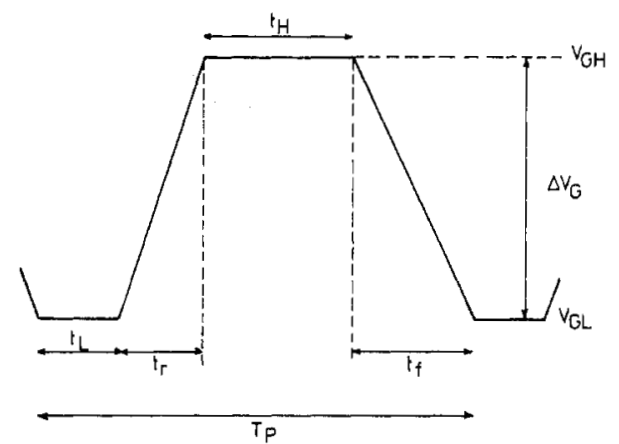

Fig. 7. Waveform applied at the gate when performing charge pumping. The different parameters are indicated on the figure.

to meet the one which is required to keep the trap occupation in dynamic equilibrium with the voltage sweep. The channel is in steady-state condition as long as [16]

$$
\left.\frac{d Q_{t}}{d t}\right|_{e m}>\left.\frac{d Q_{t}}{d t}\right|_{s s}
$$

where $d Q_{t} /\left.d t\right|_{s s}$ is the rate of change of trapped charge density, required to maintain steady-state condition, and is given by

$$
\left.\frac{d Q_{t}}{d t}\right|_{s s}=-q^{2} \overline{D_{i t}} \frac{d \psi_{s}}{d t}
$$

and $d Q_{t} /\left.d t\right|_{e m}$ is the real rate of change of trapped charge density as imposed by the emission of holes to the valence band, and is given by

$$
\left.\frac{d Q_{t}}{d t}\right|_{e m}=-q \frac{d}{d t} n_{t}(t)
$$

with $n_{t}(t)=$ carrier density (= holes) in the traps $\left(\mathrm{cm}^{-2}\right)$.

2) As soon as this rate of change of trapped charge imposed by the emission process becomes smaller than the one required by the voltage sweep at the gate (7), the channel is in the nonsteady-state regime and the emptying of the traps is controlled completely by the emission process, as described in detail by Simmons and Wei [16] and Kaden and Reimer [17] [21]. In this case the occupancy function of holes is shown to be similar in shape to the Fermi-Dirac distribution function, but is dependent on time. The transition from the steady state to the nonsteady state occurs at a certain gate voltage which can be calculated from (7) and (8). Because of the shape of the $\psi_{s}$ versus $V_{G}$ curve (Fig. 5) and the relatively high frequencies which are needed in order to measure a significant substrate current, this transition point between steady state and nonsteady state will always be very close to the flat-band voltage $V_{F B}[22]$. Indeed, from the flat-band voltage on the surface potential sweeps very fast from flat-band position to strong inversion through the depletion region, even if a reverse voltage is applied so that the quasi-Fermi level of minority carriers is dependent on time. In this depletion region, the concentration of free carriers (holes or electrons) is very small. Therefore, the time constant is only controlled by the time constant of the hole emission. Because of this high rate of change for the surface potential, the steady-state condition will 


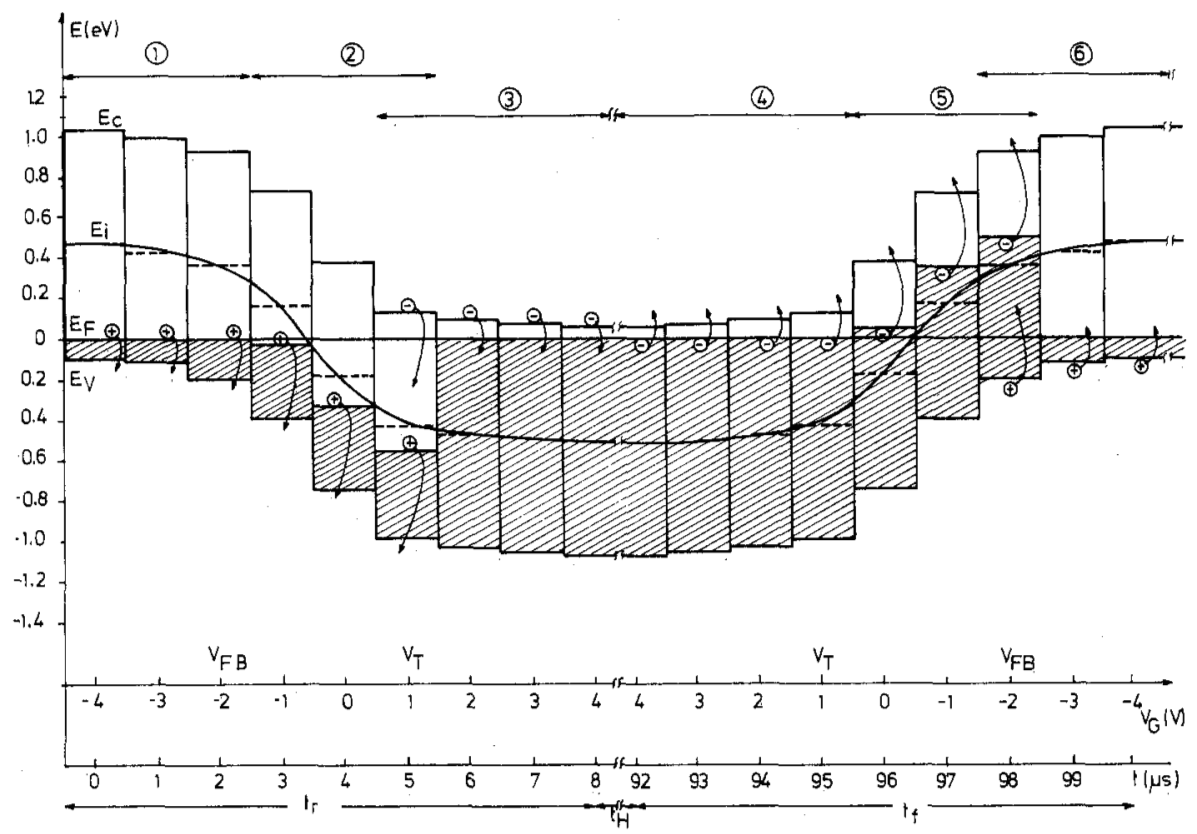

Fig. 8. Different processes occurring during one cycle of the gate pulse $\left(T_{P}=100 \mu \mathrm{s}\right)$, using the energy-band diagrams (the Fermi level is used as the zero reference level):

1) steady-state emission of holes to valence band (towards the substrate);

2) nonsteady-state emission of holes to valence band (towards the substrate);

3) trapping of electrons (from source and drain);

4) steady-state emission of electrons to conduction band (towards source and drain);

5) nonsteady-state emission of electrons to conduction band (towards source and drain); and

6) trapping of holes (from substrate)

cease very shortly after the flat-band condition so that it is quite accurate to state that from the flat-band voltage on, the channel is in the nonsteady-state regime.

3) When the gate voltage is close to the threshold voltage $V_{T}$, the trapping time constant, which is approximately given by [10]

$$
\tau_{t} \simeq \frac{1}{v_{\text {th }} \sigma_{n} n_{s}}
$$

where

$n_{s}$ is the surface concentration of minority carriers,

$v_{\text {th }}$ is the thermal velocity of the carriers, and

$\sigma_{n}$ is the capture cross section of electrons

becomes gradually smaller and consequently electrons will be trapped in the surface states not yet emptied (of holes). This process will become important when the gate voltage is almost equal to the threshold voltage. So when the gate voltage exceeds the threshold voltage, the remaining traps will be filled by electrons coming from the source and drain junctions. From that moment on, the channel is back in equilibrium with the energy bands.

When the gate is pulsed back, similar mechanisms are operating. First, electrons are emitted from the surface states and flow back to source and drain in a steady-state regime, until approximately threshold voltage is reached. Then, in the depletion region, a nonsteady-state emission of electrons from the states to the conduction band will occur followed by removal through source and drain. Finally, when the gate volt. age is approximately equal to the flat-band voltage $V_{F B}$, the trapping time constant of holes is becoming important, and holes will fill the remaining occupied (with electrons) traps.

Therefore, four different currents can be associated with these three regimes (Fig. 9(a)). If we assume that the average $D_{i t}$ value is the same in the different energy ranges of the energy gap and equal to $\overline{D_{i t}}$, these four currents can be expressed as (Fig. 9(b))

$$
\begin{aligned}
& I_{1}=-q^{2} \overline{D_{i t}} \cdot \Delta \psi_{e} \cdot f \cdot A_{G} \\
& I_{2}=q^{2} \overline{D_{i t}} \cdot \Delta \psi_{e e} \cdot f \cdot A_{G} \\
& I_{3}=-q^{2} \overline{D_{i t}} \cdot \Delta \psi_{h} \cdot f \cdot A_{G} \\
& I_{4}=q^{2} \overline{D_{i t}} \cdot \Delta \psi_{h e} \cdot f \cdot A_{G} .
\end{aligned}
$$

The net charge pumping current, measured at the substrate, is given by

$$
I_{\mathrm{SUB}}=I_{C P}=I_{4}+I_{3}=q^{2} \overline{D_{i t}}\left(\Delta \psi_{h e}-\Delta \psi_{h}\right) f \cdot A_{G} .
$$

The current measured at source and drain is given by

$$
I_{S / D}=I_{1}+I_{2}=q^{2} \overline{D_{i t}}\left(\Delta \psi_{e e}-\Delta \psi_{e}\right) f \cdot A_{G}=I_{C P} \text {. }
$$

According to the simple theory of emission of carriers from surface states, the following expressions can be derived by using a Taylor series expansion of the exponential time depen- 

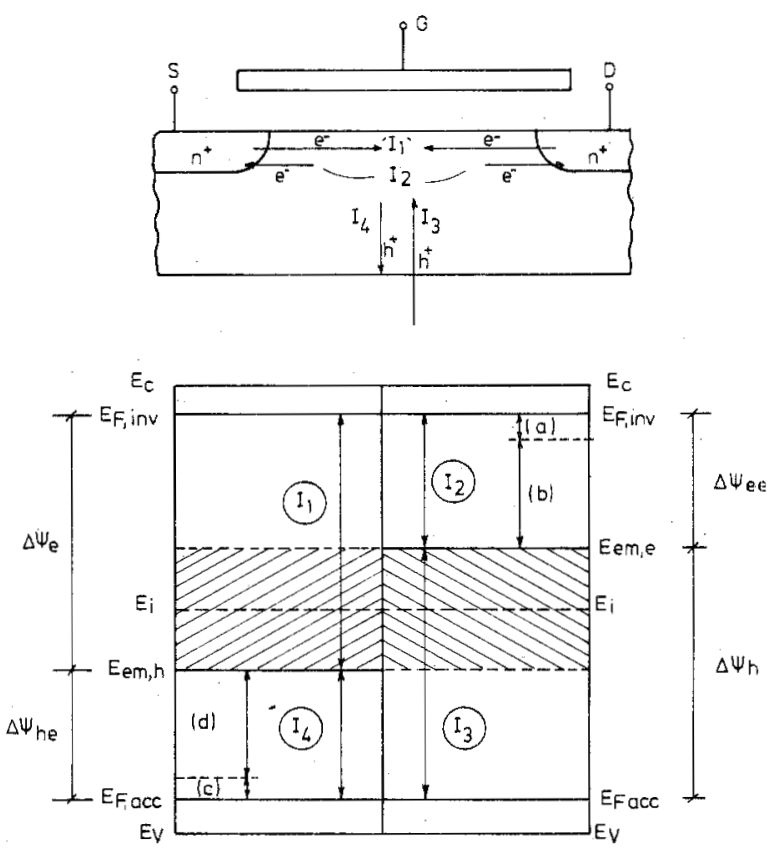

Fig. 9. Top: four different currents flowing during one cycle of the gate pulse. Bottom: different energy regions associated with these four current components: $I_{1}$ is the trapping current of elections, corresponding to $\Delta \psi_{e} ; I_{2}$ is the emission current of electrons $((1)$ is steady-state, (b) is nonsteady-state), corresponding to $\Delta \psi_{e e} ; I_{3}$ is the trapping current of holes, corresponding to $\Delta \psi_{h}$; and $I_{4}$ is the emission current of holes ((c) is steady-state, (d) is nonsteady-state), corresponding to $\Delta \psi_{h e}$. The shaded area corresponds to the net current flowing into source and drain from the substrate.

dent term in [16, eq. (31)]

$$
E_{e m, e}-E_{i}=-k T \ln \left(v_{\mathrm{th}} \cdot \sigma_{n} \cdot n_{i} \cdot t_{e m, e}+e^{\left(E_{i}-E_{F, \mathrm{inv}}\right) / k T}\right)
$$

and

$$
E_{e m, h}-E_{i}=k T \ln \left(v_{\text {th }} \cdot \sigma_{p} \cdot n_{i} \cdot t_{e m, h}+e^{\left(E_{F, \text { acc }}-E_{i}\right) / k T}\right)
$$

where $t_{e m, e}$ and $t_{e m, h}$ are the times of nonsteady-state er aission for electrons and holes, respectively, and the exponential terms are introduced to account for the case when the ernission levels are situated closer to the band edges than the quasiFermi levels.

According to the assumptions made in the previous der vation these emission times are given by

$$
\begin{aligned}
t_{e m, e} & =\frac{\left|V_{F B}-V_{T}\right|}{\left|\Delta V_{G}\right|} \cdot t_{f} \\
t_{e m, h} & =\frac{\left|V_{F B}-V_{T}\right|}{\left|\Delta V_{G}\right|} \cdot t_{r}
\end{aligned}
$$

for square pulses (see Fig. 10(a)), while for triangular wiveforms $t_{e m, e}$ and $t_{e m, h}$ are given by

$$
\begin{aligned}
& t_{e m, e}=\frac{\left|V_{F B}-V_{T}\right|}{\left|\Delta V_{G}\right|} \cdot \frac{1}{f} \cdot \alpha \\
& t_{e m, h}=\frac{\left|V_{F B}-V_{T}\right|}{\left|\Delta V_{G}\right|} \cdot \frac{1}{f} \cdot(1-\alpha)
\end{aligned}
$$

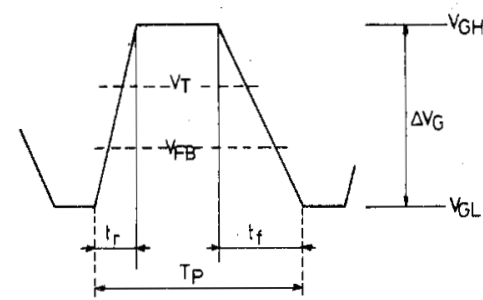

(a)

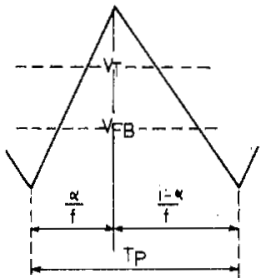

(b)
Fig. 10. Definition of $t_{f}$ and $t_{r}$ for square pulses and of $\alpha$ for triangular (or saw tooth) pulses:

where $f$ is the frequency and $\alpha$ is defined as the fraction of the period where the gate voltage is rising (Fig. 10(b)).

The charge-pumping current is then given by (for $\mathrm{n}$-channel)

$$
\begin{aligned}
I_{C P}= & 2 q \overline{D_{i t}} f \cdot A_{G} \cdot k T\left[\ln \left(v_{\text {th }} n_{t} \sqrt{\sigma_{n} \sigma_{p}}\right)\right. \\
& \left.+\ln \left(\frac{\left|V_{F B}-V_{T}\right|}{\left|\Delta V_{G}\right|} \sqrt{t_{f} \cdot t_{r}}\right)\right]
\end{aligned}
$$

for square pulses, and by

$$
\begin{aligned}
I_{C P}= & 2 q \overline{D_{i t}} f \cdot A_{G} \cdot k T\left[\ln \left(v_{\mathrm{th}} n_{i} \sqrt{\sigma_{n} \sigma_{p}}\right)\right. \\
& \left.+\ln \left(\frac{\left|V_{F B}-V_{T}\right|}{\left|\Delta V_{G}\right|} \frac{1}{f} \sqrt{\alpha \cdot(1-\alpha)}\right)\right]
\end{aligned}
$$

for sawtooth pulses.

The same formulas apply for p-channel transistors, taking into account the reverse sign for the current. These formulas are valid for charge-pumping measurements in "normal" conditions, i.e., without taking into account special effects such as the decreasing current with frequency for very high frequencies (above a few megahertz) and small inversion voltages (see Section V-E), which is due to the suppression of trapping because of too short an inversion time:

Another effect, observed at constant frequency, is the decrease of the current with decreasing inversion time duration caused by the oxide traps close to the interface, which are not filled anymore for very small pulse duration [13]. The exponential terms in (13) and (14) are also neglected, assuming that the emission levels are always closer to midgap than the Fermi levels. This is true if the fall and rise times are larger than a few nanoseconds.

The sensitivity of the charge-pumping technique can be predicted from (19) and (20). By using the values of the different parameters as determined experimentally, densities as low as $10^{9} \mathrm{~cm}^{-2} \mathrm{eV}^{-1}$ can be measured on transistors of $100-\mu \mathrm{m}^{2}$ channel area at a frequency of $100 \mathrm{kHz}$. Anyhow, the technique is much more sensitive than capacitance techniques provided that the latter could be performed on devices of the same size.

\section{EXPERIMENTAL RESULTS}

Measurements were done on p-channel as well as on $\mathrm{n}$ channel transistors. The geometrical configuration was chosen as to avoid geometric components. The transistor parameters for the different devices used in this study are summarized in 
TABLE I

Transistor Parameters

\begin{tabular}{|c|c|c|c|}
\hline & TR1 & TR2 & TR3 3 \\
\hline type & n-channel & $n$-channel & $p$-channel \\
\hline width $w$ (um) & 80 & 80 & 300 \\
\hline$I_{e f f}$ (um) & 7.7 & 80 & 19 \\
\hline$t_{o x}(\mathrm{~nm})$ & 80 & 80 & 7.2 \\
\hline $\mathrm{N}_{0}\left(\mathrm{~cm}^{-3}\right)$ & $2 \times 10^{16}$ & $2 \times 10^{16}$ & $1.1 \times 10^{15}$ \\
\hline$v_{T}(V)$ & 1.5 & 1.5 & -1 \\
\hline$v_{F B}(V)$ & -1.3 & -1.3 & -0.4 \\
\hline
\end{tabular}

Table I. In this section we will show measured data together with the theoretical predictions.

\section{A. Dependence on the Pulse Shape}

In Fig. 11 the measured and calculated frequency dependence of the charge-pumping current is shown, for both square pulses and triangular pulses with duty cycle $\alpha=0.5$ and $\alpha=0.15$. The gate voltage was switched between -4 and $+4 \mathrm{~V}$ while a reverse voltage of $0.5 \mathrm{~V}$ was applied to $S$ and $D$. It can be seen that for the square pulses a linear frequency dependence is obtained because of the fixed (i.e., frequency independent) rise and fall times which give rise to a constant amount of emitted charge per cycle, and consequently to the same recombining charge per cycle.

For the triangular pulses, however, the frequency-dependent rise and fall times also give rise to a frequency-dependent emitted charge and therefore to a frequency-dependent charge recombining with the substrate majority carriers. This is expressed in (20). Because of the larger rise and fall times for the triangular waveforms as compared to the square waveforms, the current in the former case is of course expected to be smaller than in the latter case. Changing the duty cycle $\alpha$ of the triangular pulses from 0.5 to 0.15 (which means applying sawtooth pulses), yields the result expected from the theory (20). By showing the recombined charge per cycle, which is given by

$$
Q_{s s}=\frac{I_{C P}}{f}
$$

as a function of the frequency on a semilogarithmic plot, one obtains a straight line when using triangular pulses

$$
\begin{aligned}
Q_{s s}= & 2 q \overline{D_{i t}} A_{G}: k T\left[\ln \left(v_{\mathrm{th}} n_{i} \sqrt{\sigma_{p} \sigma_{n}}\right)\right. \\
& \left.+\ln \left(\frac{\left|V_{F B}-V_{T}\right|}{\left|\Delta V_{G}\right|} \cdot \frac{1}{f} \sqrt{\alpha(1-\alpha)}\right)\right] .
\end{aligned}
$$

From the extrapolation of this curve to zero charge, the obtained frequency $f_{0}$ gives a value for the geometrical mean value of the capture cross sections. Indeed, if one puts $Q_{s s}=0$ in (22), it follows

$$
\sqrt{\sigma_{p} \sigma_{n}}=\frac{1}{v_{\mathrm{th}} \cdot n_{i}} \cdot \frac{\left|\Delta V_{G}\right|}{\left|V_{F B}-V_{T}\right|} \cdot \frac{f_{0}}{\sqrt{\alpha(1-\alpha)}} .
$$

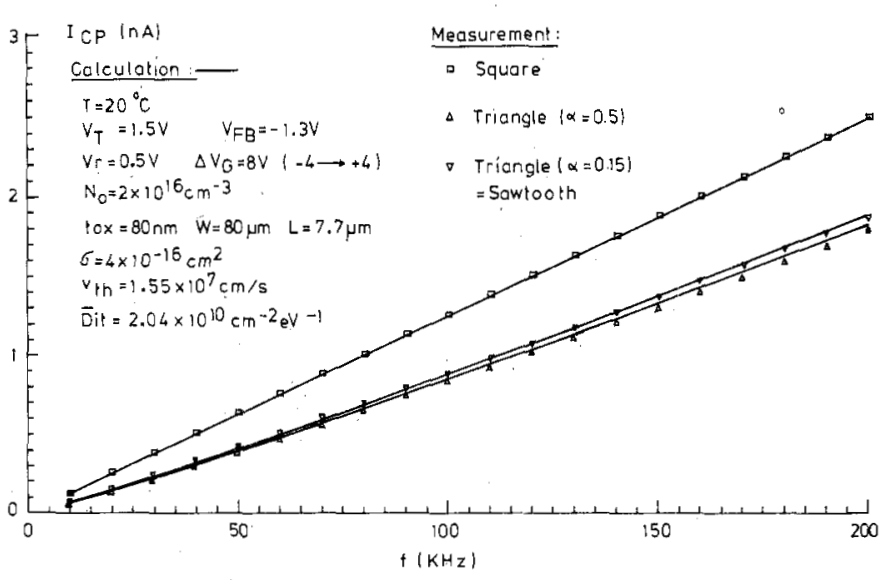

Fig. 11. Frequency dependence of the charge-pumping current for square puises and triangular pulses with $\alpha=0.5$ and $\alpha=0.15$. The symbols are measured points, while the solid lines are the calculated curves, using the proposed model.

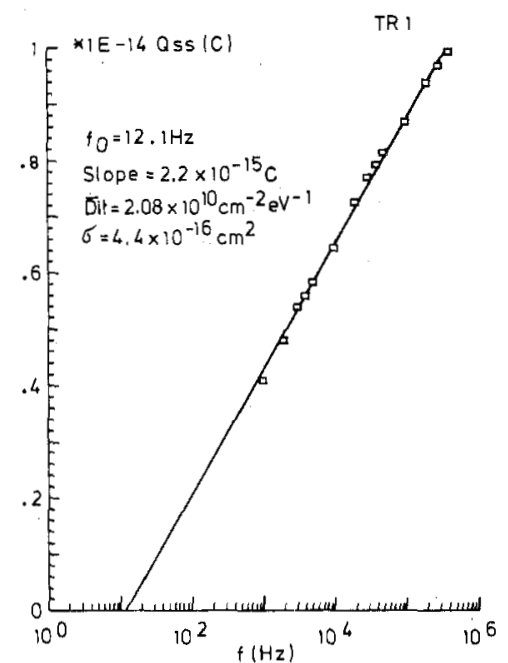

Fig. 12. $Q_{s s}$ versus frequency used to determine $\sqrt{\sigma_{n} \sigma_{p}}$ and $\overline{D_{i t}}$.

The slope of the curve is given by

$$
\frac{d Q_{s s}}{d \log f}=\frac{2 q k T \overline{D_{i t}}}{\log e} \cdot A_{G}
$$

and allows the determination of the mean value of the surfacestate density without the need for any other parameter than the temperature and the gate area. In Fig. 12 this was done for $\alpha=0.5$. A mean capture cross section of about $4.4 \times$ $10^{-16} \mathrm{~cm}^{2}$ and an average surface-state density of $2.08 \times 10^{10}$ $\mathrm{cm}^{-2}: \mathrm{eV}^{-1}$ were obtained.

This method also provides a tool for obtaining the temperature dependence of $\sigma$ if one assumes $\sigma_{p}$ and $\sigma_{n}$ to be equal. By using the $\sigma$ value obtained in this way one can fit the measured and the theoretical curve in order to obtain a good and reliable mean value of $\overline{D_{i t}}$. over that part of the forbidden bandgap which was scanned in the experiment. In Fig. 11 this fit was made and a $\overline{D_{i t}}$ value of $2.04 \times 10^{10} \mathrm{~cm}^{-2} \cdot \mathrm{eV}^{-1}$ was obtained, which corresponds to the value obtained from the slope of Fig. 12 . 


\section{B. Dependence on Gate Voltage and on Reverse Voltage at Source and Drain}

Fig. 13 shows the measured and calculated charge-pumping currents as a function of the top value $V_{G H}$ of the gate fulse and of the reverse voltage $V_{r}$ at source and drain. As one can see, the increase of charge-pumping current with gate voltage can now exactly be predicted by the emission phenomenon when considering that

$$
t_{e m, e / h}=\frac{\left|V_{F B}-V_{T}\right|}{\left|V_{G H}-V_{G L}\right|} \cdot t_{f, r}
$$

Indeed, by increasing the top level $V_{G H}$ of the gate volt ige, one obviously decreases the time that the transistor is in depletion, and consequently the time which is available for emission. Therefore there will be less emission and more recombination, and so the current will increase. The theory of emission excludes any first-order influence of surface potertial on the charge-pumping current because this influence is suppressed by the nonsteady-state emission phenomenon. The stronger increase for $V_{r}=0 \mathrm{~V}$ at voltages $V_{G H}$ of about $\leqq$ to $10 \mathrm{~V}$ is caused by the second increase in the charge-pumping current, due to the filling of traps under that part of the gate which is on top of the field oxide, as already mentionec in Section II.

By increasing the reverse voltage on the other hand, one $\lrcorner b$ tains a similar effect, but instead of decreasing the emiss on time, one increases it because of the influence of the reverse voltage on the threshold voltage due to the body effect. An increase of $V_{r}$ is accompanied by an increase of $\left|V_{F B}-V_{T}\right|$ and therefore as predicted by (25) a decrease of the chargepumping current.

Moreover, the effective gate area is modulated by increas.ng the reverse voltage by the widening of the surface space-cha:ge layers around source and drain in accumulation. When using a simple one-dimensional model, one can obtain

$$
A_{G}=W \cdot L\left(V_{r}\right)=W \cdot\left[L_{\mathrm{eff}}-2 \cdot \sqrt{\frac{2 \epsilon_{s i}}{q N_{0}}\left(V_{r}+2 \phi_{F}\right)}\right]
$$

where

$N_{0} \quad$ is the surface doping concentration and

$L_{\text {eff }}$ is the effective channel length between source and drain junctions.

When introducing both influences in the charge-pumping formulas, one obtains the calculated curve of Fig. 13, using $N_{0}:=$ $2 \times 10^{16} \mathrm{~cm}^{-3}$ which is the value we obtained also with the conventional methods to determine doping concentrations.

The measured rising edges of the curves (charge-pumping current versus $V_{G H}$ ) cannot be predicted by the formulas nor by any simple calculation of surface potential. This rising edgre is caused by the partial recombination and trapping when the surface potential reaches $2 \phi_{F}$, either by increasing the reverie voltage $V_{r}$ or by increasing the top gate voltage $V_{G H}$. As $: 1$ ready stated by Elliot [11] this rising edge contains no information about the sweep of the surface potential through the forbidden bandgap.

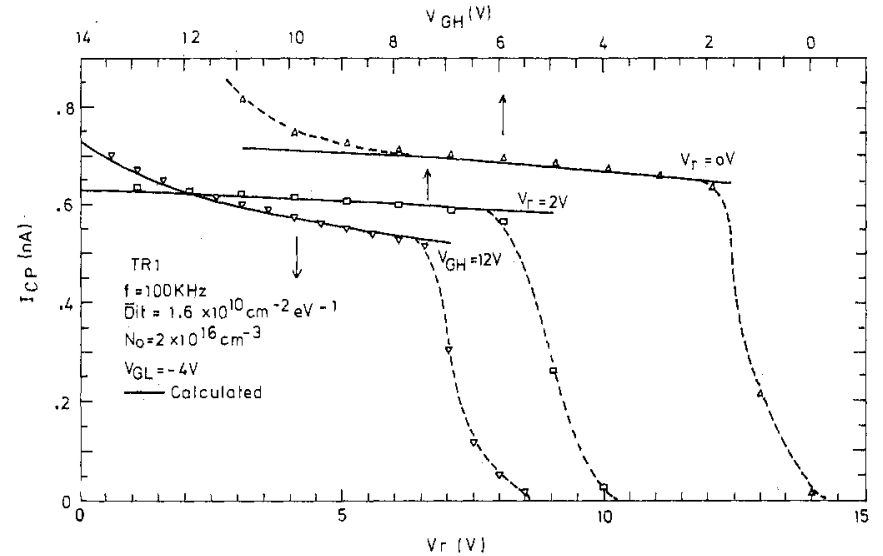

Fig. 13. Charge pumping current dependence on top level of the gate pulse (top scale) and on reverse voltage at source and drain (bottom scale). The symbols connected by a dashed line are the measured points, whereas the solid lines are the model prediction.

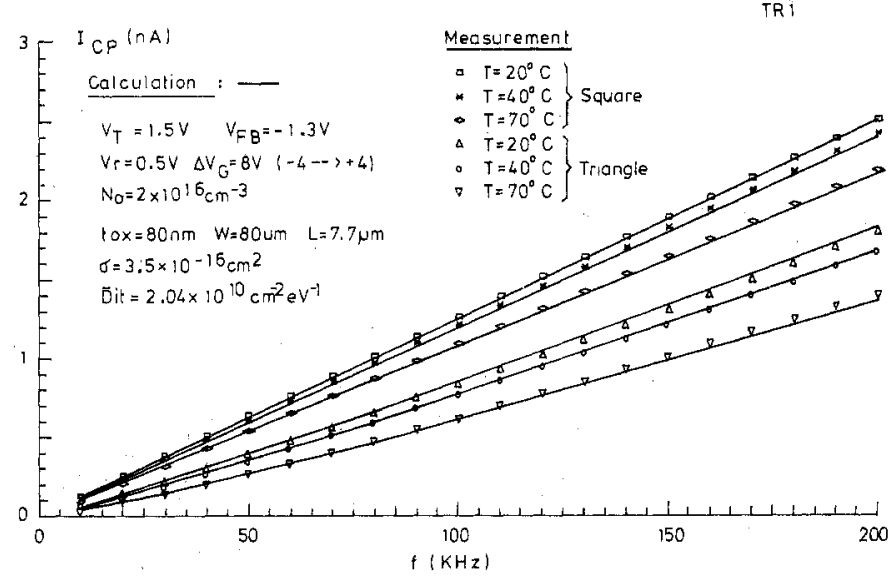

Fig. 14. Charge-pumping current versus frequency for square and triangular pulses for three different temperatures. Symbols are measured points, solid lines are calculations using the model.

\section{Dependence on Temperature}

Because of the strong temperature dependence of the emission process, the charge-pumping experiment is also very sensitive to temperature. By using (19) and (20), and after introducing the temperature dependence of all parameters, one can obtain the charge-pumping characteristics at different temperatures, as shown on Fig. 14 for both square and triangular waveforms. Because the holes and electrons are emitted more readily at higher temperatures, the current will obviously decrease with increasing temperature. As is seen from the figure, this decrease can be predicted in a very accurate and reliable manner using the presented emission theory for charge pumping.

\section{Illustration of the Geometric Current Component}

In order to illustrate the influence of a geometric component, Fig. 15 shows the curves for TR2 using method B with $W / L=1$, which is not a very favorable geometry in order to avoid the geometric current component. When using square pulses, one notices a very steep current increase with a welldefined maximum. When using triangular pulses the pheno- 


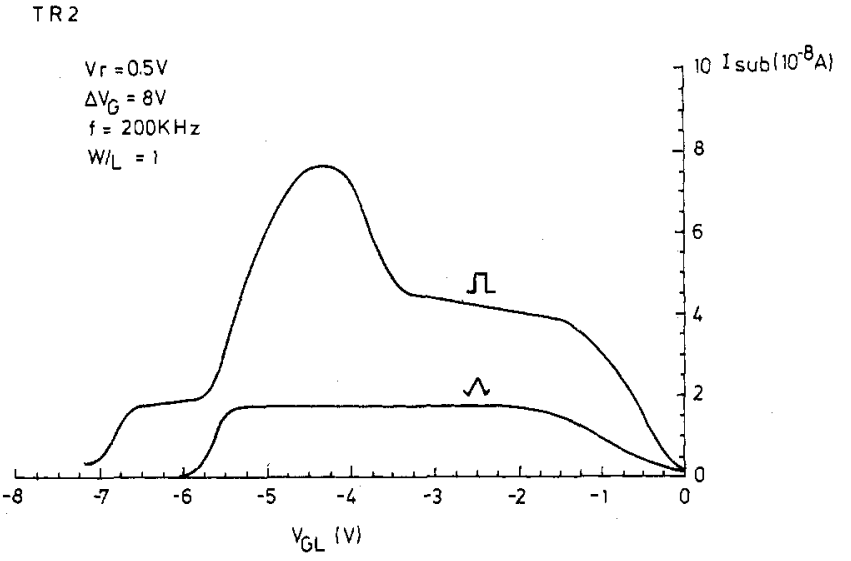

Fig. 15. Elliot curves for triangular and square gate pulses on a square geometry transistor $(W / L=1)$. The geometric component is eliminated when using sawtooth pulses.

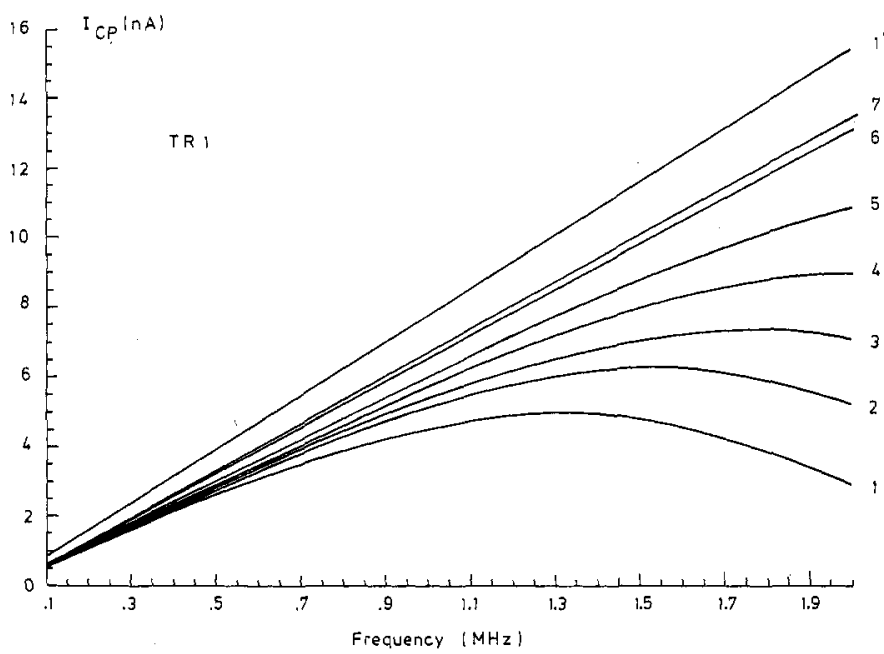

Fig. 16. Charge pumping current versus frequency. Curve $1^{\prime}$ is for square pulses; curves 1 to 7 are for triangular pulses with $V_{G H}$ increasing from $V_{T}$ (curve 1) to $V_{T}+4 \mathrm{~V}$ (curve 7). Influence of trapping time constant.

menon vanishes and the current saturates as expected. This demonstrates clearly the totally different behavior of a geometric component as opposed to the normal recombination of interface trapped charge, and at the same time provides a way to decide whether a geometric current component is present. It was found that in all of our transistors with $W / L>1$ and for any value of $L$ used, a geometric component is neither present nor required to explain the observed charge-pumping current behavior. In contradiction with earlier publications [10], [15], it is concluded that the charge-pumping technique is not restricted to short-channel devices.

\section{E. Influence of Trapping Time Constant}

As was already mentioned in Subsection III-C, the time during which the channel is in inversion should be long enough to allow the carriers to be trapped. When the inversion time becomes smaller than the trapping time constant of the surface states, they will no longer be completely filled and the chargepumping current will decrease. This is illustrated in Fig. 16, where the charge-pumping current is shown versus frequency for different types of applied gate waveforms. The upper curve $1^{\prime}$ is for square pulses. It can be seen that even at $2 \mathrm{MHz}$ the current is still linearly increasing. Indeed, the inversion time is constant and about equal to half the pulse period (250 ns for $2 \mathrm{MHz}$ ), which is still sufficiently high to allow complete filling of the states. The curves 1 to 7 are for triangular waveforms, where the top level of the gate pulse $V_{G H}$ is increasing from a few millivolts above threshold (curve 1) to $4 \mathrm{~V}$ above threshold (curve 7). By doing so, the time the gate voltage is above threshold becomes very small (order of $1 \mathrm{~ns}$ ) for high frequencies in the case of curve 1, but is increasing with increasing $V_{G H}$ (curves 2-7). As a result the traps can no longer be totally filled in the case of curve 1 and the cur. rent is decreasing with increasing frequency.

\section{Determination of Interface State Distribution}

From the previous considerations one can conclude that introducing the emission of carriers in a nonsteady-state regime both to the valence and conduction band makes the technique of charge pumping into a very reliable and quantitatively accurate method to determine interface states directly on an MOS transistor. However, it is often desirable to obtain an interface state distribution over the forbidden energy gap. So far, only a mean value for $D_{i t}$ was obtained from charge-pumping experiments.

Attempts to determine the surface-state distribution from charge-pumping measurements have been reported. Brugler and Jespers [10] already suggested determining the surfacestate density distribution from the rising edge of the current versus gate voltage characteristic, using the surface potential versus gate voltage dependence. However, this procedure was found to be incorrect by Elliot [11], since during the rising edge of this characteristic, the potential barrier between source and drain and the substrate prevents the carriers from flowing into the channel to fill the empty states. Elliot suggested using the rising edge of his current versus gate pulse base level in order to obtain the surface-state distribution. For this purpose he also needed the dependence of surface potential on gate voltage, which he obtained by measuring the low-frequency $C-V$ characteristic of the MOS structure. However, in view of the phenomenon of emission, this method is equally invalid, for the same reasons as those mentioned earlier. Indeed, as long as the channel remains in the depletion regime, the ma. jority carriers of the substrate cannot recombine with the trapped carriers in the surface states because of the potential barrier between the substrate and the surface. Only when the surface potential reaches the flat-band condition will trapping occur and the filled states can recombine with holes. This means that the rising edge of the current versus pulse base level only gives information in a very small region of a few kilotesla around the flat-band position, and not over the whole range from flat-band to inversion. It will be shown now that by taking into account the emission process, one can obtain the surface-state distribution over a large part of the forbidden energy gap by doing a simple experiment and without needing the dependence of surface potential on gate voltage.

When applying square pulses with variable fall times while keeping the rise time constant, one scans the energy range in 
the upper half of the bandgap between the conduction band and midgap. On the other hand, when varying the rise time while keeping the fall time constant, the energy states in the lower half of the bandgap are scanned. The charge which recombines during each cycle can be written as

$$
Q_{s s}=q \cdot A_{G} \int_{E_{1}}^{E_{2}} D_{i t}(E) d E
$$

where $E_{1}$ and $E_{2}$ are the boundaries of the energy range which is scanned and $D_{i t}(E)$ is the interface state density at energy $E$.

The derivative of $Q_{s s}$ with respect to the fall or rise time $t_{i}$ of the pulse is given by

$$
\frac{d Q_{s s}}{d t_{i}}=q A_{G}\left[D_{i t}\left(E_{2}\right) \frac{d E_{2}}{d t_{i}}-D_{i t}\left(E_{1}\right) \frac{d E_{1}}{d t_{i}}\right] \text {. }
$$

When keeping the rise time constant, for example, while clanging the fall time, one obtains

$$
\frac{d Q_{s s}}{d t_{f}}=q A_{G} D_{i t}\left(E_{2}\right) \frac{d E_{2}}{d t_{f}}
$$

since $E_{1}$ is independent of fall time.

According to (13) $E_{2}$ can be written as

$$
E_{2}=E_{i}-k T \ln \left[v_{\mathrm{th}} \sigma_{n} n_{i} \frac{\left|V_{F B}-V_{T}\right|}{\left|\Delta V_{G}\right|} \cdot t_{f}\right]
$$

and so

$$
\frac{d E_{2}}{d t_{f}}=-\frac{k T}{t_{f}}
$$

Therefore, according to (29) while taking into account $(31)$, $D_{i t}\left(E_{2}\right)$ can be found from

$$
D_{i t}\left(E_{2}\right)=-\frac{t_{f}}{q A_{G} k T} \frac{d Q_{s s}}{d t_{f}}
$$

or since $Q_{s s}=I_{C P} / f, D_{i t}\left(E_{2}\right)$ is given by

$$
D_{i t}\left(E_{2}\right)=-\frac{t_{f}}{q A_{G} k T f} \frac{d I_{C P}}{d t_{f}} .
$$

By keeping the fall time constant and changing the rise lime one obtains in a similar way

$$
D_{i t}\left(E_{1}\right)=-\frac{t_{r}}{q A_{G} k T f} \frac{d I_{C P}}{d t_{r}} .
$$

By measuring the charge pumping current with variable fall and rise times (e.g., using an HP8010A pulse generator) consecutively, one can easily obtain the energy distribution of the surface states in a large part of the forbidden energy yap, whereby the temperature can be an extra variable.

In this derivation it is assumed that the capture cross jections for electrons and holes are energy independent, which is probably not correct [23]. However, around the middle: of the energy gap these capture cross sections are almost energy independent, and therefore the obtained energy dependenco of $D_{i t}$ is reliable in this region. A more elaborate study on the determination of the energy distribution of the interface stittes with the charge-pumping technique will be published elsewhere [22].
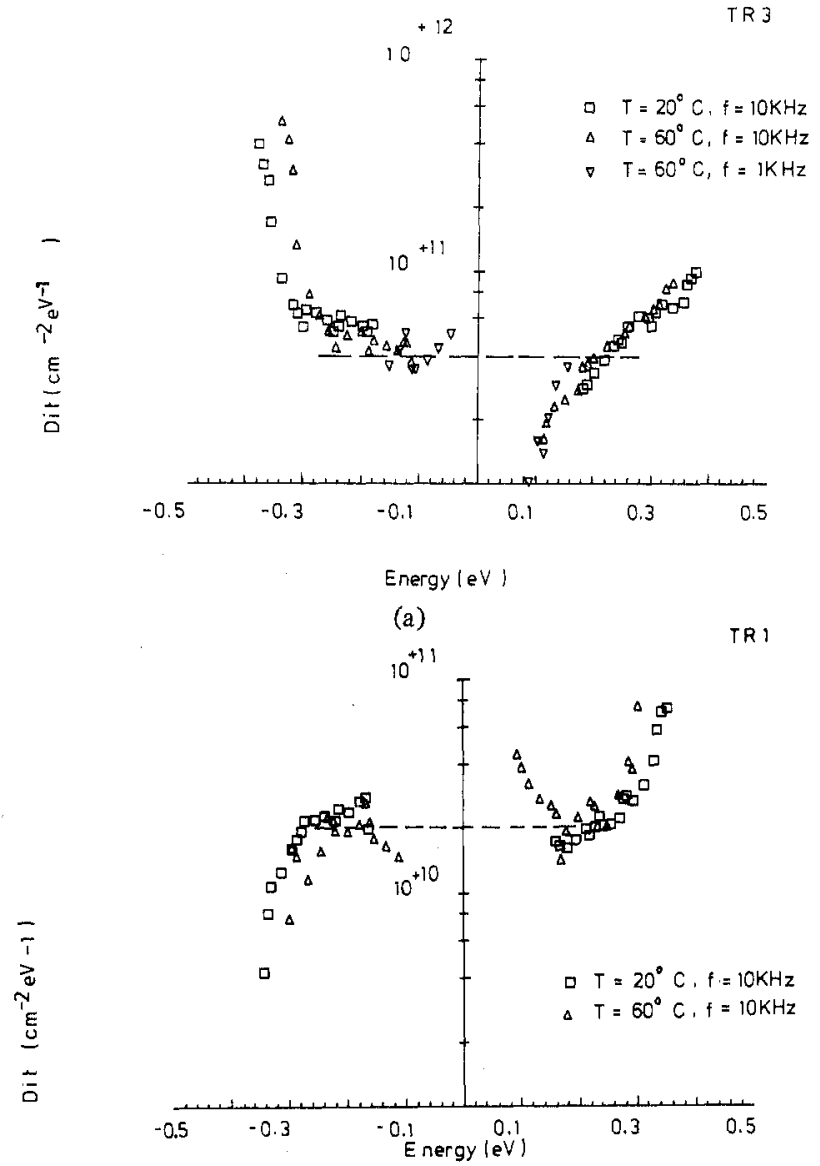

(b)

Fig. 17. Energy distribution of interface states as determined by the new method for (a) a p-channel transistor and (b) an n-channel transistor. The dashed horizontal line is the mean value $\overline{D_{i t}}$ obtained using the method discussed in Section V (the intrinsic level is used as the zero reference level).

As an example of the proposed method, Fig. 17(a) and (b) shows the energy distribution of $D_{i t}$ as obtained on $\mathrm{p}$-channel and n-channel transistors, respectively. The distributions were obtained using two different temperatures $\left(20\right.$ and $60^{\circ} \mathrm{C}$ ) and with two different frequencies ( 1 and $10 \mathrm{kHz}$ ), in order to reach as broad an energy range as possible. By decreasing the frequency one can measure closer towards midgap because the maximum fall and rise times increase. By increasing the tem. perature, deeper levels can also be reached because of the temperature dependence of the emission process. On the figures the mean value $\overline{D_{i t}}$ obtained according to the method explained in Section $\mathrm{V}$ is also shown.

\section{CONCLUSIONS}

In this paper we presented a model for charge pumping which describes and explains all the phenomena which were not well understood in the past, taking into account the nonsteady-state emission of holes and electrons to the valence and conduction band, respectively, depending on the charge state of the $\mathrm{Si}-\mathrm{SiO}_{2}$ interface. This model is based on the more general description of the phenomenon by Kaden and Reimer [17] $-[21]$, but uses some assumptions and approximations to the more exact theory in order to make the analysis more 
tractable. In this way the charge-pumping technique becomes a very powerful tool and a reliable quantitative method for determining interface state densities directly on MOS transistors. All of the inherent problems which were described in the past have been solved or removed, and the geometric component which was often invôked by other investigators in order to explain their seemingly peculiar results was shown to be present only in a few transistors with unfavorable geometry.

Moreover, a simple technique was derived from this theory to obtain the energy distribution of interface states without knowing the dependence of surface potential on gate voltage. Our simple but accurate model is sufficiently reliable to make the charge-pumping technique into a standard technique for measuring the $\mathrm{Si}-\mathrm{SiO}_{2}$ interface characteristics on MOS transistors with a sensitivity better than that of other existing techniques using capacitors and without having their disadvantages.

\section{REFERENCES}

[1] M. Kuhn, "A quasi-static technique for MOS C-V and surface state measurements," Solid-State Electron., vol. 13, p. 873, 1970.

[2] E. H. Nicollian and A. Goetzberger, "The $\mathrm{Si}_{-} \mathrm{SiO}_{2}$ interfaceElectrical properties as determined by the metal-insulator-silicon conductance technique," Bell Syst. Tech. J., vol. 46, p. 1055, 1967.

[3] L. M. Terman, "An investigation of surface states at a $\mathrm{Si}-\mathrm{SiO}_{2}$ interface employing MOS diodes," Solid-State Electron., vol. 5 , p. 285,1962 .

[4] P. V. Gray and D. M. Brown, "Density of $\mathrm{Si}^{-\mathrm{SiO}_{2}}$ interface states," Appl. Phys. Lett., vol. 8, p. 31, 1966.

[5] N. Beltrán, G. Groeseneken, R. F. De Keersmaecker, and H. E. Maes, "A reliable approach to charge pumping in MOS transistors," presented at ESSDERC-SSDT, Munich, Sept. 1982.

[6] R. J. Van Overstraeten, G. Declerck, and P. A. Muls, "Theory of the MOS transistor in weak inversion," IEEE Trans. Electron Devices, vol. ED-22, p. 282, 1975.

[7] K. L. Wang and A. O. Evwaraye, "Determination of interface and bulk trap states of IGFET's using deep-level transient spectroscopy," J. Appl. Phys., vol. 47, p. 4574, 1976.

[8] F. M. Klaassen, "Characterization of low $1 / f$ noise in MOS transistors," IEEE Trans. Electron Devices, vol. ED-18, p. 887, 1971.
[9] G. Broux, R. Van Overstraeten, and G. Declerck, "Experimental results on fast surface states and $1 / f$ noise in MOS transistors," Electron. Lett., vol. 11, p. 97, 1975.

[10] J. S. Brugler and P.G.A. Jespers, "Charge pumping in MOS devices," IEEE Trans. Electron Devices, vol. ED-16, p. 297, 1969.

[11] A.B.M Elliot, "The use of charge pumping currents to measure surface state densities in MOS transistors," Solid-State Electron., vol. 19, p. $241,1976$.

[12] J. Bosch, thesis, Tech. Phys. Lab., Groningen State University, Groningen, Germany, 1979.

[13] M. Declercq and P. Jespers. "Analysis of interface properties in MOS transistors by means of charge pumping measurements," Rev. HF, Acta Techn. Belgica, vol. 9, p. 244, 1974.

[14] N. Beltrán, "Characterization of interface trapped charge in MOS transistors using charge pumping technique," M.S. thesis, Katholieke Universiteit Leuven, Heverlee, Belgium, 1981.

[15] W. V. Backensto and C. R. Viswanathan, "Measurement of interface state characteristics of MOS transistor $u$ tilising charge pumping techniques," Proc. IEE, vol. 128, pt. 1, no. 2, p. 44, 1981.

[16] J. G. Simmons and L. S. Wei, "Theory of dynamic charge current and capacitance characteristics in MIS systems containing distributed surface traps," Solid-State Electron., vol. 16, p. 53, 1973.

[17] G. Kaden and H. Reimer, "Ladungsträgereinfang in MOS-Strukturen mit externer Minoritätsträgerquelle," Fhys. Status Solidi, vol. 32, p. $183,1975$.

[18] G. Kaden and H. Reimer, "Bestimmung der Dichte und des Einfangquerschnittes von Oberflächenzuständen mit dem Oberflächenladungspumpeffekt," Phys. Status Solidi, vol. 44, p. K117, 1977.

[19] G. Kaden and H. Reimer, "Impulsstromverhalten von MOSStrukturen mit zum Substrat kurzgeschlossenem externem pnÜbergang bei linearer Gatespannungsansteuerung," Phys. Status Solidi, vol. 52, p. 35, 1979.

[20] -MOS-Strukturen mit sperrseitig vorgespanntem externem pn-C̈bergang bei linearer Gatespannungsansteuerung-Impulsstromverhalten," Phys. Status Solidi, vol. 53, p. 195, 1979.

[21] —-, "MOS-Strukturen mit sperrseitig vorgespanntem externem pn-Úbergang bei linearer Gatespannungsansteuerung-Bestimmung von $N_{\mathrm{st}}$ und $\sigma$," Phys. Status Solidi, vol. 59, p. 719, 1980.

[22] G. Groeseneken, H. E. Maes, N. Beltrán, and R. F. De Keersmaecker, in Proc. INFOS'83, Eindhoven, North Holland, 1983, to be published.

[23] A. Goetzberger, E. Klausmann, and M. J. Schulz, "Interface states on semiconductor/insulator surfaces," CRC Crit. Rev. Solid-State Sci., vol. 6, p. 1, 1976. 